\title{
S3-Leitlinie: Diagnostik und Therapie biliärer Karzinome
}

\author{
Kurzversion 2.00 - Juni 2021, AWMF-Registernummer: 032-0530L
}

Autoren

M. Bitzer ${ }^{1}$, S. Voesch ${ }^{1}$, J. Albert ${ }^{2}$, P. Bartenstein ${ }^{3}$, W. Bechstein ${ }^{4}$, S. Blödt ${ }^{5}$, T. Brunner ${ }^{6}$, F. Dombrowski ${ }^{7}$, M. Evert ${ }^{8}$, M. Follmann ${ }^{9}$, C. La Fougère ${ }^{10}$, P. Freudenberger ${ }^{11}$, A. Geier ${ }^{12}$, E. Gkika ${ }^{13}$, M. Götz ${ }^{14}$, E. Hammes ${ }^{15}$, T. Helmberger ${ }^{16}$, R. T. Hoffmann ${ }^{17}$, W. P. Hofmann ${ }^{18}$, P. Huppert ${ }^{19}$, A. Kautz ${ }^{20}$, G. Knötgen ${ }^{21}$, J. Körber ${ }^{22}$, D. Krug ${ }^{23}$, F. Lammert ${ }^{24}$, H. Lang ${ }^{25}$, T. Langer ${ }^{26}$, P. Lenz ${ }^{27}$, A. Mahnken ${ }^{28}$, A. Meining ${ }^{29}$, O. Micke ${ }^{30}$, S. Nadalin ${ }^{31}$, H. P. Nguyen ${ }^{32}$, J. Ockenga ${ }^{33}$, K. Oldhafer $^{34}$, P. Paprottka ${ }^{35}$, K. Paradies ${ }^{36}$, P. Pereira ${ }^{37}$, T. Persigehl ${ }^{38}$, M. Plauth ${ }^{39}$, R. Plentz ${ }^{40}$, J. Pohl ${ }^{41}$, J. Riemer ${ }^{42}$, P. Reimer ${ }^{43}$, J. Ringwald ${ }^{44}$, U. Ritterbusch ${ }^{45}$, E. Roeb ${ }^{46}$, B. Schellhaas ${ }^{47}$, P. Schirmacher ${ }^{48}$, I. Schmid ${ }^{49}$, A. Schuler ${ }^{50}$, D. von Schweinitz ${ }^{51}$, D. Seehofer ${ }^{52}$, M. Sinn ${ }^{53}$, A. Stein ${ }^{54}$, A. Stengel ${ }^{55}$, N. Steubesand ${ }^{56}$, C. Stoll ${ }^{57}$, A. Tannapfel ${ }^{58}$, A. Taubert ${ }^{59}$, J. Trojan ${ }^{60}$, I. van Thiel ${ }^{61}$, R. Tholen ${ }^{62}$, A. Vogel ${ }^{63}$, T. Vogl ${ }^{64}$, H. Vorwerk ${ }^{65}$, F. Wacker ${ }^{66}$, O. Waidmann ${ }^{67}$, H. Wedemeyer ${ }^{68}$, H. Wege ${ }^{69}$, D. Wildner ${ }^{70}$, C. Wittekind ${ }^{71}$, M. A. Wörns ${ }^{72}$, P. Galle ${ }^{72}$, N. Malek ${ }^{73}$

Institute

1 Medizinische Klinik I, Universitätsklinikum Tübingen

2 Abteilung für Gastroenterologie, Hepatologie und Endokrinologie, Robert-Bosch-Krankenhaus, Stuttgart

3 Klinik und Poliklinik für Nuklearmedizin, LMU Klinikum, München

4 Klinik für Allgemein-, Viszeral-, Transplantations- und Thoraxchirurgie, Universitätsklinikum Frankfurt

5 AWMF-Geschäftsstelle, Berlin

6 Klinik für Strahlentherapie, Universitätsklinikum Magdeburg

7 Institut für Pathologie, Universitätsmedizin Greifswald

8 Institut für Pathologie, Regensburg

9 Office des Leitlinienprogrammes Onkologie, c/o Deutsche Krebsgesellschaft e.V., Berlin

10 Nuklearmedizin und Klinische Molekulare Bildgebung, Tübingen

11 Clinical Guideline Services GmbH, Berlin

12 Medizinische Klinik und Poliklinik II, Universitätsklinikum Würzburg

13 Klinik für Strahlenheilkunde, Department für Radiologische Diagnostik und Therapie, Universitätsklinikum Freiburg

14 Kliniken Böblingen

15 Lebertransplantierte Deutschland e. V., Ansbach

16 Institut für Radiologie, Neuroradiologie und minimal-invasive Therapie, München Klinik Bogenhausen, München

17 Institut und Poliklinik für Diagnostische und Interventionelle Radiologie, Dresden

18 Gastroenterologie am Bayerischen Platz, medizinisches Versorgungszentrum, Berlin

19 Radiologisches Zentrum, Max Grundig Klinik, Bühl

20 Deutsche Leberhilfe e.V., Köln

21 Konferenz onkologischer Kranken- und Kinderkrankenpflege, Hamburg

22 Klinik Nahetal, Fachklinik für onkologische Rehabilitation und Anschlussrehabilitation, Bad Kreuznach

23 Klinik für Strahlentherapie, Universitätsklinikum Schleswig-Holstein, Kiel

24 Medizinische Hochschule Hannover
25 Klinik für Allgemein-, Viszeral und Transplantationschirurgie, Universitätsmedizin der Johannes Gutenberg-Universität Mainz

26 Office des Leitlinienprogrammes Onkologie, c/o Deutsche Krebsgesellschaft e.V., Berlin

27 Universitätsklinikum Münster, Zentrale Einrichtung Palliativmedizin, Münster

28 Klinik für Diagnostische und Interventionelle Radiologie, Universitätsklinikum Gießen und Marburg GmbH, Marburg

29 Medizinische Klinik und Poliklinik II des Universitätsklinikums Würzburg

30 Klinik für Strahlentherapie und Radioonkologie, Franziskus Hospital Bielefeld

31 Universitätsklinik für Allgemein-, Viszeral- und Transplantationschirurgie, Universitätsklinikum Tübingen

32 Humangenetik, Ruhr-Universität, Bochum

33 Medizinische Klinik II, Klinikum Bremen-Mitte, Bremen

34 Klinik für Leber-, Gallenwegs- und Pankreaschirurgie, Semmelweis Universität, Asklepios Campus Hamburg

35 Abteilung für interventionelle Radiologie, Klinikum rechts der Isar der Technischen Universität München

36 Konferenz onkologischer Kranken- und Kinderkrankenpflege, Hamburg

37 Abteilung für interventionelle Radiologie, Klinikum rechts der Isar der Technischen Universität München

38 Institut für Diagnostische und Interventionelle Radiologie, Universitätsklinikum Köln

39 Städtisches Klinikum Dessau

40 Klinikum Bremen-Nord, Innere Medizin, Bremen

41 Interventionelles Endoskopiezentrum und Schwerpunkt Gastrointestinale Onkologie, Asklepios Klinik Altona, Hamburg

42 Lebertransplantierte Deutschland e. V., Bretzfeld

43 Institut für diagnostische und interventionelle Radiologie, Städtisches Klinikum Karlsruhe gGmbH, Karlsruhe

44 Psychosomatische Medizin und Psychotherapie, Universitätsklinikum Tübingen

45 Hospizarbeit am Universitätsklinikum Essen 
46 Medizinische Klinik II, Universitätsklinikum Gießen und Marburg GmbH, Gießen

47 Medizinische Klinik I, Friedrich-Alexander Universität Erlangen-Nürnberg, Erlangen

48 Pathologisches Institut, Universitätsklinikum Heidelberg

49 Zentrum Pädiatrische Hämatologie und Onkologie,

Dr. von Haunersches Kinderspital, Klinikum der Universität München

50 Medizinische Klinik, Alb Fils Kliniken GmbH, Göppingen

51 SRH Wilhelm Löhe Hochschule, Fürth

52 Klinik und Poliklinik für Viszeral-, Transplantations-, Thorax- und Gefäßchirurgie, Universitätsklinikum Leipzig

53 Medizinische Klinik II, Universitätsklinikum HamburgEppendorf

54 Hämatologisch-Onkologischen Praxis Eppendorf, Hamburg

55 Psychosomatische Medizin und Psychotherapie,

Universitätsklinikum Tübingen

56 Clinical Guideline Services GmbH, Kiel

57 Klinik Herzoghöhe Bayreuth, Bayreuth

58 Institut für Pathologie der Ruhr-Universität Bochum am Berufsgenossenschaftlichen Universitätsklinikum Bergmannsheil, Bochum

59 Kliniksozialdienst, Universitätsklinikum Heidelberg, Bochum

60 Medizinische Klinik I, Universitätsklinikum Frankfurt, Frankfurt am Main

61 Deutsche Leberhilfe e.V., Köln

62 Deutscher Verband für Physiotherapie e. V., Köln

63 Klinik für Gastroenterologie, Hepatologie, Endokrinologie der Medizinischen Hochschule Hannover, Hannover

64 Universitätsklinikum Frankfurt, Institut für Diagnostische und Interventionelle Radiologie, Frankfurt

65 Klinik für Strahlentherapie, Universitätsklinikum Gießen und Marburg GmbH, Marburg
66 Institut für Diagnostische und Interventionelle Radiologie der Medizinischen Hochschule Hannover, Hannover

67 Medizinische Klinik I, Universitätsklinikum Frankfurt, Frankfurt am Main

68 Klinik für Gastroenterologie, Hepatologie und Endokrinologie Medizinische Hochschule Hannover, Hannover

69 Medizinische Klinik und Poliklinik, Universitätsklinikum Hamburg-Eppendorf, Hamburg

70 Innere Medizin, Krankenhäuser Nürnberger Land GmbH, Lauf an der Pegnitz

71 Institut für Pathologie, Universitätsklinikum Leipzig, Leipzig

72 Medizinische Klinik und Poliklinik, Universitätsklinikum Mainz, Mainz

73 Medizinische Klinik I, Universitätsklinikum Tübingen, Tübingen

\section{Bibliografie}

Z Gastroenterol 2022; 60: 219-238

DOI $10.1055 / a-1589-7638$

ISSN 0044-2771

(C) 2022. Thieme. All rights reserved.

Georg Thieme Verlag KG, Rüdigerstraße 14,

70469 Stuttgart, Germany

\section{Korrespondenzadresse}

Sabrina Voesch

Medizinische Klinik I

Universitätsklinikum Tübingen, Otfried-Müller-Str. 10,

72076 Tübingen, Deutschland

Sabrina.Voesch@med.uni-tuebingen.de

Nisar Malek

Medizinische Klinik I

Universitätsklinikum Tübingen, Otfried-Müller-Str. 10, 72076 Tübingen, Deutschland

\begin{tabular}{|l|l|l|}
\hline \multicolumn{2}{|l|}{ Inhaltsverzeichnis } & Seite \\
\hline 1. & Informationen zu dieser Leitlinie & 221 \\
\hline 1.1. & Herausgeber & 221 \\
\hline 1.2. & Federführende Fachgesellschaft(en) & 221 \\
\hline 1.3. & Finanzierung der Leitlinie & 221 \\
\hline 1.4. & Kontakt & 221 \\
\hline 1.5. & Zitierweise & 221 \\
\hline 1.6. & Besonderer Hinweis & 221 \\
\hline 1.7. & Ziele des Leitlinienprogramms Onkologie & 222 \\
\hline 1.8. & Weitere Dokumente zu dieser Leitlinie & 222 \\
\hline 1.9. & Zusammensetzung der Leitliniengruppe & 222 \\
\hline 1.9 .1$. & Koordination und Redaktion & 222 \\
\hline 1.9 .2$. & Beteiligte Fachgesellschaften und Organisationen & 222 \\
\hline 1.9 .3$. & Patientenbeteiligung & 222 \\
\hline 1.9 .4$. & Methodische Begleitung & 222 \\
\hline & & \\
\hline
\end{tabular}

\begin{tabular}{|l|l|l|}
\hline \multicolumn{2}{|l|}{ Inhaltsverzeichnis } & Seite \\
\hline 1.10. & Verwendete Abkürzungen & 224 \\
\hline 2. & Einführung & 226 \\
\hline 2.1. & Geltungsbereich und Zweck & 226 \\
\hline 2.1 .1$. & Zielsetzung und Fragestellung & 226 \\
\hline 2.1 .2$. & Adressaten & 226 \\
\hline 2.1 .3$. & Gültigkeitsdauer und Aktualisierungsverfahren & 227 \\
\hline 2.1 .4$. & Grundlagen der Methodik & 227 \\
\hline 2.1 .5$. & $\begin{array}{l}\text { Schema der Evidenzgraduierung nach Oxford } \\
\text { (Version 2011) }\end{array}$ & 227 \\
\hline 2.1 .6$. & Schema der Empfehlungsgraduierung & 227 \\
\hline 2.1 .7$. & Statements & 227 \\
\hline 2.1 .8$. & Expertenkonsens (EK) & 227 \\
\hline 2.1 .9$. & Klug entscheiden & 227 \\
\hline 2.1 .10$. & Unabhängigkeit und Darlegung möglicher & 227 \\
\hline & Interessenkonflikte & \\
\hline & & \\
\hline
\end{tabular}




\begin{tabular}{|c|c|c|}
\hline \multicolumn{2}{|c|}{ Inhaltsverzeichnis } & \multirow{2}{*}{$\begin{array}{l}\text { Seite } \\
229\end{array}$} \\
\hline 3. & $\begin{array}{l}\text { Diagnostik und Thearpie des Hepatozellulären } \\
\text { Karzinoms }\end{array}$ & \\
\hline 4. & Diagnostik und Therapie der biliären Karzinome & 230 \\
\hline 4.1 . & Risikofaktoren, Prävention und Früherkennung & 230 \\
\hline 4.1.1. & Risikofaktoren & 230 \\
\hline 4.1.2. & $\begin{array}{l}\text { Prophylaktische und therapeutische Maßnahmen } \\
\text { zur Reduktion des Risikos der Entstehung } \\
\text { von biliären Karzinomen }\end{array}$ & 230 \\
\hline 4.1.3. & Früherkennung & 231 \\
\hline 4.2. & Histopathologische und molekulare Diagnostik & 231 \\
\hline 4.3. & Bildgebende und endoskopische Diagnostik & 232 \\
\hline 4.3.1. & Endoskopische Diagnostik & 232 \\
\hline 4.4 . & Operative und interventionelle Therapieverfahren & 232 \\
\hline 4.4.1. & Resektion & 232 \\
\hline 4.4.2. & Lebertransplantation & 233 \\
\hline 4.4.3. & Interventionelle Therapieverfahren & 233 \\
\hline 4.4.4. & Endoskopische Therapieverfahren & 233 \\
\hline 4.4.5. & Stereotaxie & 235 \\
\hline 4.4.6. & Nachsorge & 235 \\
\hline 4.5 . & Systemtherapie & 235 \\
\hline 4.5.1. & Adjuvante Therapie & 235 \\
\hline 4.5.2. & Neoadjuvante Therapie primär resektabler Tumoren & 235 \\
\hline 4.5.3. & Systemtherapie lokal fortgeschrittener Tumoren & 235 \\
\hline 4.5.4. & Palliative Systemtherapie & 236 \\
\hline 4.5.5. & Verlaufskontrollen unter Systemtherapie & 236 \\
\hline 4.6. & $\begin{array}{l}\text { Supportivtherapie des Hepatozellulären Karzinoms } \\
\text { und der biliären Karzinome }\end{array}$ & 236 \\
\hline 4.6.1. & Ernährung & 236 \\
\hline 4.6.2. & Palliativmedizinische Behandlung beim $\mathrm{HCC} / \mathrm{CCA}$ & 236 \\
\hline 4.6.3. & Rehabilitation, Sport- und Bewegungstherapie & 237 \\
\hline 4.6.4. & Psychoonkologie & 237 \\
\hline 5. & Qualitätsindikatoren & 237 \\
\hline 6. & Abbildungsverzeichnis & 237 \\
\hline 7. & Tabellenverzeichnis & 237 \\
\hline \multicolumn{2}{|c|}{ Literaturverzeichnis } & 237 \\
\hline
\end{tabular}

\section{Informationen zu dieser Leitlinie}

\subsection{Herausgeber}

Leitlinienprogramm Onkologie der Arbeitsgemeinschaft der Wissenschaftlichen Medizinischen Fachgesellschaften e. V. (AWMF), Deutschen Krebsgesellschaft e. V. (DKG) und Deutschen Krebshilfe (DKH)

\subsection{Federführende Fachgesellschaft(en)}

Deutsche Gesellschaft für Gastroenterologie, Verdauungs- und Stoffwechselkrankheiten

\section{DGVS \\ Deutsche Gesellschaft für \\ Gastroenterologie, \\ Verdauungs- und \\ Stoffwechselkrankheiten}

\subsection{Finanzierung der Leitlinie}

Diese Leitlinie wurde von der Deutschen Krebshilfe im Rahmen des Leitlinienprogramms Onkologie gefördert.

\subsection{Kontakt}

Office Leitlinienprogramm Onkologie

c/o Deutsche Krebsgesellschaft e. V.

Kuno-Fischer-Straße 8

14057 Berlin

leitlinienprogramm@krebsgesellschaft.de

www.leitlinienprogramm-onkologie.de

\subsection{Zitierweise}

Leitlinienprogramm Onkologie (Deutsche Krebsgesellschaft, Deutsche Krebshilfe, AWMF): Diagnostik und Therapie des Hepatozellulären Karzinoms und biliärer Karziome, Langversion 2.0, Juni 2021, AWMF Registernummer: 032/-0530L, https://www.leitlinienpro gramm-onkologie.de/leitlinien/hepatozellulaeres-karzinom-hcc/

\subsection{Besonderer Hinweis}

Die Medizin unterliegt einem fortwährenden Entwicklungsprozess, sodass alle Angaben, insbesondere zu diagnostischen und therapeutischen Verfahren, immer nur dem Wissensstand zur Zeit der Drucklegung der Leitlinie entsprechen können. Hinsichtlich der angegebenen Empfehlungen zur Therapie und der Auswahl sowie Dosierung von Medikamenten wurde die größtmögliche Sorgfalt beachtet. Gleichwohl werden die Benutzer aufgefordert, die Beipackzettel und Fachinformationen der Hersteller zur Kontrolle heranzuziehen und im Zweifelsfall einen Spezialisten zu konsultieren. Fragliche Unstimmigkeiten sollen bitte im allgemeinen Interesse der OL-Redaktion mitgeteilt werden.

Der Benutzer selbst bleibt verantwortlich für jede diagnostische und therapeutische Applikation, Medikation und Dosierung.

In dieser Leitlinie sind eingetragene Warenzeichen (geschützte Warennamen) nicht besonders kenntlich gemacht. Es kann also aus dem Fehlen eines entsprechenden Hinweises nicht geschlossen werden, dass es sich um einen freien Warennamen handelt.

Das Werk ist in allen seinen Teilen urheberrechtlich geschützt. Jede Verwertung außerhalb der Bestimmung des Urhebergesetzes ist ohne schriftliche Zustimmung der OL-Redaktion unzulässig und strafbar. Kein Teil des Werkes darf in irgendeiner Form ohne schriftliche Genehmigung der OL-Redaktion reproduziert werden. Dies gilt insbesondere für Vervielfältigungen, Übersetzungen, Mikroverfilmungen und die Einspeicherung, Nutzung und Verwertung in elektronischen Systemen, Intranets und dem Internet. 
In dieser Leitlinie wird aus Gründen der Lesbarkeit die männliche Form verwendet, nichtsdestoweniger beziehen sich die Angaben auf Angehörige aller Geschlechter.

\subsection{Ziele des Leitlinienprogramms Onkologie}

Die Arbeitsgemeinschaft der Wissenschaftlichen Medizinischen Fachgesellschaften e. V., die Deutsche Krebsgesellschaft e. V. und die Deutsche Krebshilfe haben sich mit dem Leitlinienprogramm Onkologie $(\mathrm{OL})$ das Ziel gesetzt, gemeinsam die Entwicklung und Fortschreibung und den Einsatz wissenschaftlich begründeter und praktikabler Leitlinien in der Onkologie zu fördern und zu unterstützen. Die Basis dieses Programms beruht auf den medizinisch-wissenschaftlichen Erkenntnissen der Fachgesellschaften und der DKG, dem Konsens der medizinischen Fachexperten, Anwender und Patienten sowie auf dem Regelwerk für die Leitlinienerstellung der AWMF und der fachlichen Unterstützung und Finanzierung durch die Deutsche Krebshilfe. Um den aktuellen Stand des medizinischen Wissens abzubilden und den medizinischen Fortschritt zu berücksichtigen, müssen Leitlinien regelmäßig überprüft und fortgeschrieben werden. Die Anwendung des AWMF-Regelwerks soll hierbei Grundlage zur Entwicklung qualitativ hochwertiger onkologischer Leitlinien sein. Da Leitlinien ein wichtiges Instrument der Qualitätssicherung und des Qualitätsmanagements in der Onkologie darstellen, sollten sie gezielt und nachhaltig in den Versorgungsalltag eingebracht werden. So sind aktive Implementierungsmaßnahmen und auch Evaluationsprogramme ein wichtiger Bestandteil der Förderung des Leitlinienprogramms Onkologie. Ziel des Programms ist es, in Deutschland professionelle und mittelfristig finanziell gesicherte Voraussetzungen für die Entwicklung und Bereitstellung hochwertiger Leitlinien zu schaffen. Diese hochwertigen Leitlinien dienen nicht nur dem strukturierten Wissenstransfer, sondern können auch in der Gestaltung der Strukturen des Gesundheitssystems ihren Platz finden. Zu erwähnen sind hier evidenzbasierte Leitlinien als Grundlage zum Erstellen und Aktualisieren von Disease-Management-Programmen oder die Verwendung von aus Leitlinien extrahierten Qualitätsindikatoren im Rahmen der Zertifizierung von Organtumorzentren.

\subsection{Weitere Dokumente zu dieser Leitlinie}

Bei diesem Dokument handelt es sich um die Kurzversion der S3-Leitlinie „Diagnostik und Therapie des hepatozellulären Karzinoms und biliärer Karzinome“. Neben der Kurzversion wird es folgende ergänzende Dokumente zu dieser Leitlinie geben:

- Langversion der Leitlinie

- Laienversion (Patientenleitlinie)

- Leitlinienreport zum Erstellungsprozess der Leitlinie

- Evidenztabellen

Diese Leitlinie und alle Zusatzdokumente sind über die folgenden Seiten zugänglich.

- Leitlinienprogramm Onkologie (https://www.leitlinienpro gramm-onkologie.de/leitlinien/hepatozellulaeres-karzinom-hcc/)

- AWMF (www.leitlinien.net)

- Deutsche Gesellschaft für Gastroenterologie, Verdauungs- und Stoffwechselkrankheiten (www.dgvs.de)

- Guidelines International Network (www.g-i-n.net).
Die Leitlinie ist außerdem in der App des Leitlinienprogramms Onkologie enthalten.

Weitere Informationen unter: https://www.leitlinienprogrammonkologie.de/app/

\subsection{Zusammensetzung der Leitliniengruppe}

\subsubsection{Koordination und Redaktion}

Prof. Dr. Nisar P. Malek

Ärztlicher Direktor Medizinische Klinik Universitätsklinikum Tübingen Prof. Dr. Michael Bitzer

Stellvertretender Ärztlicher Direktor Medizinische Klinik Universitätsklinikum Tübingen

Prof. Dr. Peter R. Galle

Ärztlicher Direktor Universitätsmedizin der Johannes GutenbergUniversität Mainz

Sabrina Voesch

Ärztin in Weiterbildung Medizinische Klinik Universitätsklinikum Tübingen

\subsubsection{Beteiligte Fachgesellschaften und Organisationen}

In $\triangleright$ Tab. 1 sind die an der Leitlinienerstellung beteiligten medizinischen Fachgesellschaften und sonstigen Organisationen sowie deren mandatierte Vertreter aufgeführt.

Außerdem wurden folgende Fachgesellschaften für den Leitlinienprozess angeschrieben, diese haben jedoch keinen Mandatsträger benannt:

- Deutsche Gesellschaft für Allgemeinmedizin und Familienmedizin

- Deutsche Gesellschaft für Pflegewissenschaft e. V.

- Arbeitsgemeinschaft Pädiatrische Onkologie

- Arbeitsgemeinschaft Onkologische Thoraxchiurgie

- Deutsche Gesellschaft für Ernährung e. V.

- Deutsche Gesellschaft für Klinische Chemie und Laboratoriumsmedizin

Die Zuordnung der beteiligten Mandatsträger und Experten finden Sie in den $>$ Tab. 2, 3

\subsubsection{Patientenbeteiligung}

Die Leitlinie wurde unter direkter Beteiligung von vier Patientenvertretern erstellt.

Herr Ingo van Thiel, Herr Achim Kautz und Frau Elke Hammes waren von Beginn an in die Erstellung der Leitlinie eingebunden und nahmen mit eigenem Stimmrecht an der Konsensuskonferenz teil. Herr Kautz war der Stellvertreter von Herrn Thiel und hat daher nicht abgestimmt. Frau Riemer ersetzte Frau Hammes ab der Videokonsensuskonferenz 08/2020.

\subsubsection{Methodische Begleitung}

1. Durch das Leitlinienprogramm Onkologie:

a) Dr. med. Markus Follmann, MPH, MSc

(OL Office c/o Deutsche Krebsgesellschaft)

b) Thomas Langer, Dipl. Soz. Wiss.

(OL Office c/o Deutsche Krebsgesellschaft) 
- Tab. 1 Beteiligte Fachgesellschaften und Organisationen.

\section{Beteiligte Fachgesellschaften und Organisationen}

Deutsche Gesellschaft für Allgemein- und Viszeralchirurgie (DGAV)

Deutsche Gesellschaft für Chirurgie (DGCH)

Deutsche Gesellschaft für Ernährungsmedizin (DGEM)

Deutsche Gesellschaft für Endoskopie und bildgebende Verfahren (DGE-BV)

Deutsche Gesellschaft für Hämatologie und Onkologie (DGHO)

Deutsche Gesellschaft für Innere Medizin (DGIM)

Deutsche Gesellschaft für interventionelle Radiologie und minimalinvasive Therapie (DeGIR)

Deutsche Gesellschaft für Nuklearmedizin (DGN)

Deutsche Gesellschaft für Pädiatrische Onkologie und Hämatologie (GPOH)

Deutsche Gesellschaft für Palliativmedizin (DGPall)

Deutsche Gesellschaft für Pathologie (DGP)

Berufsverband deutscher Pathologen (BDP)

Deutsche Gesellschaft für Radioonkologie (DEGRO)

Deutsche Gesellschaft für Ultraschall in der Medizin (DEGUM)

Deutscher Verband für Physiotherapie (ZKV)

Deutsche Röntgengesellschaft e. V. (DRG)

Deutsche Gesellschaft für Gastroenterologie, Verdauungs- und Stoffwechselkrankheiten (DGVS)

Deutsche Transplantationsgesellschaft (DTG)

Deutsche Gesellschaft für Humangenetik (GfH)

Deutsche Leberhilfe e. V.

Deutsche Leberstiftung

Lebertransplantierte Deutschland e. V.

Arbeitsgemeinschaft Internistische Onkologie (AIO)

Arbeitsgemeinschaft Onkologische Pathologie

Arbeitsgemeinschaft Radiologische Onkologie (ARO)

Arbeitsgemeinschaft Tumorklassifikation in der Onkologie (ATO)

Arbeitsgemeinschaft Supportive Maßnahmen in der Onkologie (AGSMO)

Arbeitsgemeinschaft Onkologische Rehabilitation und Sozialmedizin (AGORS)

Chirurgische Arbeitsgemeinschaft Onkologie (CAO-V)

Arbeitsgemeinschaft Psychoonkologie (PSO)

Konferenz Onkologischer Kranken- und Kinderkrankenpflege (KOK)

Arbeitsgemeinschaft Palliativmedizin (APM)

Arbeitsgemeinschaft Bildgebung in der Onkologie (ABO)

Arbeitsgemeinschaft Prävention und Integrative Medizin in der Onkologie (AG PRIO)

Arbeitsgemeinschaft Soziale Arbeit in der Onkologie (ASO)

\section{Mandatsträger}

Oldhafer K., Seehofer D. (Stellv.)

Lang $\mathrm{H}$.

Plauth M., Ockenga J. (Stellv.)

Meining A.

Sinn M.

Bitzer M., Trojan J.

Pereira P., Mahnken A. (Stellv.), Huppert P. (Stellv.)

Bartenstein P., La Fougère Ch. (Stellv.)

Schmid I., v. Schweinitz D. (Stellv.)

Lenz $\mathrm{P}$.

Schirmacher P., Evert M.

Schirmacher P., Evert M.

Vorwerk H., Krug D. (Stellv.)

Schuler A., Wildner D. (Stellv.)

Tholen R.

Vogl T., Paprottka P., Wacker F., Helmberger T.

Malek N., Galle P., Götz M., Lammert F., Plentz R.

Nadalin S.

Nguyen H. P.

van Thiel I., Kautz A. (Stellv.)

Schirmacher $P$.

Hammes E. bis 10.08 .2020

Riemer J. ab 11.08.2020

Vogel A., Wege $\mathrm{H}$.

Dombrowski F.

Brunner T., Gkika E. (Stellv.)

Tannapfel A., Wittekind Ch. (Stellv.)

Stein A.

Körber J.

Bechstein W.

Stengel A., Ringwald J. (Stellv.)

Knötgen G., Paradies K. (Stellv.)

Ritterbusch U.

Persigehl T.

Stoll Ch., Micke O. (Stellv.)

Taubert A.
2. Durch die Arbeitsgemeinschaft der Wissenschaftlichen Medizinischen Fachgesellschaften e. V.:

a) Dr. rer. medic. Susanne Blödt, MScPH (AWMF-IMWI)

3. Durch die Firma Clinical Guideline Service - User Group:

b) Dr. Paul Freudenberger 
- Tab. 2 Arbeitsgruppen und deren Mitglieder.

\section{Arbeitsgruppe}

AG I. Risikofaktoren, Prävention und Früherkennung

AG II.I Histopathologische und molekulare Diagnostik

AG II.II Bildgebende Diagnostik

AG III.I Operative und interventionelle Therapieverfahren

AG III.II Systemtherapie

AG IV Supportivtherapie
Mitglieder der Arbeitsgruppe (AG-Leiter hervorgehoben)

Trojan J. , Schuler A., van Thiel I., Kautz A., Wedemeyer H., Lammert F., Roeb E., Geier A., Wildner D., Hofmann, W.P., Schmid I.

Schirmacher P. , Nguyen H.P., Dombrowski F., Evert M., Tannapfel A.

Götz M. , Paprottka P., Persigehl T., Vogl T. J., Meining A., Wacker F., Pohl J., Reimer P., Schellhaas B.

Nadalin S., Pereira P. , Bechstein W., Oldhafer K., Lang H., Bartenstein P., Vorwerk H., Brunner T., Hammes E., Vogel A., Helmberger T., Seehofer D., La Fougère Ch., Albert J., Hoffmann R.-T., Mahnken A., Huppert P., Gkika E., Krug D.

Bitzer M., Galle P. , Sinn M., Stein A., Plentz R., Schmid I., Wörns M.-A., Wege H.

Lenz P. , Ritterbusch U., Tholen R., Körber J., Stoll Ch., Taubert A., Stengel A., Knötgen G., Plauth M., Waidmann $\mathrm{O}$.

- Tab. 3 Beteiligte Experten.

\section{Beteiligte Experten}

Wedemeyer $\mathrm{H}$.

Roeb E.

Geier A.

Pohl J.

Reimer P.

Schellhaas B.

Albert J.

Hoffmann R.-T.

Hofmann W. P.

Wörns M.-A.

Waidmann O.

\section{Arbeitsgruppe}

AG I Risikofaktoren, Prävention und Früherkennung

AG I Risikofaktoren, Prävention und Früherkennung

AG I Risikofaktoren, Prävention und Früherkennung

AG II. II Bildgebende Diagnostik

AG II. II Bildgebende Diagnostik

AG II. II Bildgebende Diagnostik

AG III.I. Operative und interventionelle Therapieverfahren

AG III.I. Operative und interventionelle Therapieverfahren

AG I Risikofaktoren, Prävention und Früherkennung

AG III.II. Systemtherapie

AG IV. Supportivtherapie

\subsection{Verwendete Abkürzungen}

\begin{tabular}{|c|c|c|c|}
\hline Abkürzung & Erläuterung & Abkürzung & Erläuterung \\
\hline 3D-CRT & Three-dimensional Conformal Radiation Therapy & ATG & Antithymozytenglobulin \\
\hline $5-\mathrm{FU}$ & 5-Fluorouracil & AUC & Area under the Curve \\
\hline AASLD & American Association for the Study of Liver Diseases & AWMF & Arbeitsgemeinschaft der Wissenschaftlichen \\
\hline ACG & American College of Gastroenterology & & Medizinischen Fachgesellschaften e. V. \\
\hline AFIP & Armed Forces Institute of Pathology & $\ddot{A Z Z Q}$ & Ärztliches Zentrum für Qualität in der Medizin \\
\hline AFP & Alpha-Fetoprotein & BÄK & Bundesärztekammer \\
\hline AFP-L3 & Lektin reaktives Alpha-Fetoprotein & BCLC & Barcelona Clinic Liver Cancer \\
\hline AG & Arbeitsgruppe & Bds & Beiderseits \\
\hline ALT & Alanin-Aminotransferase & BillN & Biliäre intraepitheliale Neoplasie \\
\hline APASL & Asian Pacific Association for the Study of the Liver & BMI & Body-Mass-Index \\
\hline APRI & AST/Thrombozyten-Ratio-Index & BSC & Best Supportive Care \\
\hline ARFI & Acoustic Radiation Force Impulse Imaging & BSG & British Society of Gastroenterology \\
\hline AST & Aspartat-Aminotransferase & CA 19-9 & Carbohydrate-Antigen 19-9 \\
\hline
\end{tabular}




\begin{tabular}{|c|c|}
\hline Abkürzung & Erläuterung \\
\hline CCA & Cholangiokarzinom \\
\hline iCCA & Intrahepatisches Cholangiokarzinom \\
\hline $\mathrm{dCCA}$ & Distales Cholangiokarzinom \\
\hline eCCA & Extrahepatisches Cholangiokarzinom \\
\hline $\mathrm{pCCA}$ & Perihiläres Cholangiokarzinom \\
\hline$C D$ & Cluster of Differentiation \\
\hline CEUS & Kontrastmittel-Ultraschall \\
\hline $\mathrm{Cl}$ & Konfidenzintervall \\
\hline CIPN & Chemotherapie-induzierte periphere Neuropathie \\
\hline CLIP & Cancer of the Liver Italian Program \\
\hline $\mathrm{CNI}$ & Calcineurininhibitor \\
\hline CR & Complete Remission \\
\hline CT & Computertomografie \\
\hline CTCEA & Common Terminology Criteria for Adverse Events \\
\hline CTLA-4 & Cytotoxic T-lymphocyte-associated Protein 4 \\
\hline CU-HCC & Chinese University-HCC (Risikoscore) \\
\hline CUP & Cancer of Unkown Primary \\
\hline DAAD & Direct-acting antiviral Drugs \\
\hline DCP & des-Gamma-Carboxyprothrombin \\
\hline $\mathrm{DGCH}$ & Deutsche Gesellschaft für Chirurgie \\
\hline DGEM & Deutsche Gesellschaft für Ernährungsmedizin \\
\hline DGVS & $\begin{array}{l}\text { Deutsche Gesellschaft für Gastroenterologie, } \\
\text { Verdauungs- und Stoffwechselkrankheiten }\end{array}$ \\
\hline $\mathrm{DHC}$ & Ductus Choledochus \\
\hline DKG & Deutsche Krebsgesellschaft e. V. \\
\hline DKH & Deutsche Krebshilfe e. V. \\
\hline EASL & European Association for the Study of the Liver \\
\hline ECOG & Eastern Cooperative Oncology Group \\
\hline EG & $\begin{array}{l}\text { Empfehlungsgrad, } A=\text { starke Empfehlung, } B=\text { Empfeh- } \\
\text { lung, } 0 \text { = offene Empfehlung }\end{array}$ \\
\hline EK & Expertenkonsens \\
\hline ELTR & European Liver Transplant Registry \\
\hline EORTC & $\begin{array}{l}\text { European Organisation for Research and Treatment } \\
\text { of Cancer }\end{array}$ \\
\hline $\begin{array}{l}\text { EORTIC- } \\
\text { QLQ }\end{array}$ & $\begin{array}{l}\text { European Organisation for Research and Treatment } \\
\text { of Cancer - Quality of Life Questionnaire }\end{array}$ \\
\hline EQD2 & Equivalenzdosis von 2 Gy \\
\hline $\mathrm{ERC}(\mathrm{P})$ & Endoskopische retrograde Cholangio(pankreatiko)grafie \\
\hline ESCULAP & $\begin{array}{l}\text { Erlanger Synopsis for Contrast-enhanced Ultrasound } \\
\text { for Liver Lesion Assessment in Patients at Risk }\end{array}$ \\
\hline ESMO & European Society of Medical Oncology \\
\hline ETC & Extended Toronto Criteria \\
\hline EUS-(FNA) & Endosonografischer Ultraschall - (Feinnadelaspiration) \\
\hline FACT-H & Functional Assessment of Cancer Therapy- Hepatobiliary \\
\hline FDG-PET & Fluordeoxyglukose-Positronen-Emissionstomografie \\
\hline FIB-4 & Fibrosis-4 \\
\hline
\end{tabular}

\begin{tabular}{|c|c|}
\hline Abkürzung & Erläuterung \\
\hline GAG-HCC & $\begin{array}{l}\text { Guide with Age, Gender, HBV DNA, Core Promoter } \\
\text { Mutations and Cirrhosis-HCC }\end{array}$ \\
\hline GB-CA & Gallenblasenkarzinom \\
\hline GCP & Good Clinical Practice \\
\hline Gd-DTPA & Gadolinium-Diethylene-Triamine Pentaacetic Acid \\
\hline $\begin{array}{l}\text { Gd-EOB- } \\
\text { DTPA }\end{array}$ & $\begin{array}{l}\text { Gadolinium-Ethoxybenzyl-Diethylentriamin-Penta- } \\
\text { Essigsäure }\end{array}$ \\
\hline GEKID & $\begin{array}{l}\text { Gesellschaft der epidemiologischen Krebsregister } \\
\text { in Deutschland e.V. }\end{array}$ \\
\hline G-I-N & Guidelines International Network \\
\hline GLOBOCAN & Global Cancer Incidence, Mortality and Prevalence \\
\hline GOT & Glutamat-Oxalacetat-Transaminase \\
\hline GPT & Glutamat-Pyruvat-Transaminase \\
\hline $\mathrm{HAl}$ & Hepatische arterielle Infusion \\
\hline $\mathrm{HBsAg}$ & Hepatitis-B-surface-Antigen \\
\hline HBeAg & Hepatitis-B-envelope-Antigen \\
\hline HBV & Hepatitis B \\
\hline $\mathrm{HCC}$ & Hepatozelluläres Karzinom \\
\hline $\mathrm{HCV}$ & Hepatitis C \\
\hline HepPar1 & Hepatocyte Paraffin 1 \\
\hline $\mathrm{HR}$ & Hazard Ratio \\
\hline HSP70 & Hitzeschockprotein 70 \\
\hline hTERT & Human Telomerase Reverse Transcriptase \\
\hline ICCR & International Collaboration on Cancer Reporting \\
\hline ICD & $\begin{array}{l}\text { International Statistical Classification of Diseases } \\
\text { and Related Health Problems }\end{array}$ \\
\hline IGRT & Image Guided Radiotherapy \\
\hline ILCA & International Liver Cancer Association \\
\hline IPMN & Intraduktale papillär-muzinösen Neoplasie \\
\hline IRE & Irreversible Elektroporation \\
\hline IQWiG & $\begin{array}{l}\text { Institut für Qualität und Wirtschaftlichkeit } \\
\text { im Gesundheitswesen }\end{array}$ \\
\hline ITT & Intention to Treat \\
\hline JIS & Japan Integrated Staging Score \\
\hline KASL & Korean Association for the Study of the Liver \\
\hline LA & Leitlinienadaptation \\
\hline LAASL & $\begin{array}{l}\text { Latin American Association for the Study of the Liver } \\
\text { (LAASL) }\end{array}$ \\
\hline LiMax & Maximum liver function capacity \\
\hline LIRADS & Liver Imaging Reporting and Data System \\
\hline LL & Leitlinie \\
\hline LoE & Level of Evidence \\
\hline LTx & Lebertransplantation \\
\hline MARS & Molecular-Adsorbent-Recirculating-System \\
\hline MCN & Muzinös-zystische Neoplasie \\
\hline MRCP & Magnetresonanz-Cholangiopankreatikografie \\
\hline $\operatorname{MR}(T)$ & Magnetresonanz(tomografie) \\
\hline
\end{tabular}




\begin{tabular}{|c|c|}
\hline Abkürzung & Erläuterung \\
\hline MWA & Mikrowellenablation \\
\hline NAFLD & Non-alcoholic fatty Liver Disease \\
\hline $\mathrm{NASH}$ & Nichtalkoholische Steatohepatitis \\
\hline $\mathrm{NCl}$ & National Cancer Institute \\
\hline NCCN & The National Comprehensive Cancer Network \\
\hline NGC & National Guideline Clearinghouse \\
\hline NICE & National Institute for Health and Clinical Excellence \\
\hline NRS & Nutrition Risk Screening \\
\hline NT & Nicht transplantabel \\
\hline $\mathrm{OL}$ & Leitlinienprogramm Onkologie \\
\hline OP & Operation \\
\hline ORN & Osteoradionekrose \\
\hline OS & Overall Survival \\
\hline PBC & Primär biliäre Zirrhose \\
\hline PBD & Präoperative biliäre Drainage \\
\hline PD & Progressive Disease \\
\hline PDT & Photodynamische Therapie \\
\hline $\mathrm{PEI} / \mathrm{PAI}$ & Perkutane Ethanol-Injektion \\
\hline PET & Positronen-Emissions-Tomografie \\
\hline PFS & Progression-free Survival \\
\hline $\mathrm{PICO}$ & Population Intervention Comparison Outcome \\
\hline PR & Partial Remission \\
\hline PS & Progressive Disease \\
\hline PSC & Primär sklerosierende Cholangitis \\
\hline PV & Portalvene \\
\hline PZK & Patientenzentrierte Kommunikation \\
\hline QI & Qualitätsindikatoren \\
\hline QoL & Quality of Life \\
\hline RFA & Radiofrequenzablation \\
\hline iRFA & Intraduktale Radiofrequenzablation \\
\hline RILD & Radiation induced Liver Disease \\
\hline $\mathrm{RR}$ & Relatives Risiko \\
\hline SBRT & Stereotactic Body Radiotherapy \\
\hline SD & Stable Disease \\
\hline SEOM & Spanish Society of Medical Oncology \\
\hline SEMS & Selbstexpandierender Metallstent \\
\hline SGA & Subjective Global Assessment \\
\hline SGB & Sozialgesetzbuch \\
\hline$S R$ & Systematische Recherche \\
\hline STIKO & Ständige Impfkomission \\
\hline SVR & Substained Virological Response \\
\hline TACE & Transarterielle Chemoembolisation \\
\hline DEB-TACE & Drug-eluting Bead TACE \\
\hline TARE & Transarterielle Radioembolisation \\
\hline TNM & Tumor Nodus Metastase \\
\hline
\end{tabular}

\begin{tabular}{|l|l|}
\hline Abkürzung & Erläuterung \\
\hline TTD & Time to Deterioration \\
\hline TTP & Time to Progression \\
\hline UICC & Union for International Cancer Control \\
\hline UCSF & University of California, San Francisco \\
\hline UNOS & United Network of Organ Sharing \\
\hline US & Ultraschall \\
\hline VEGF(R) & Vascular Endothelial Growth Factor (Receptor) \\
\hline WHO & World Health Organisation \\
\hline
\end{tabular}

\section{Einführung}

\subsection{Geltungsbereich und Zweck}

\subsubsection{Zielsetzung und Fragestellung}

Die interdisziplinäre S3-Leitlinie ist ein Instrument, um die Diagnostik und Therapie des hepatozellulären Karzinoms (HCC), des Cholangiokarzinoms (CCA) und des Gallenblasenkarzinoms zu verbessern. Fachgruppen aller Disziplinen, die Patienten mit chronischen Lebererkrankungen, Verdacht auf oder bereits diagnostizierten hepatobiliäre Tumoren ambulant und/oder stationär behandeln, sollen durch die Leitlinie unterstützt werden. Die Leitlinie soll dazu beitragen, eine angemessene Gesundheitsversorgung dieser Patientengruppen sicherzustellen. Es ist weiterhin die Aufgabe der Leitlinie, betroffenen Patienten angemessene, wissenschaftlich begründete und aktuelle Verfahren in der Diagnostik, Therapie und Rehabilitation anzubieten. Dies gilt sowohl für lokal begrenzte oder lokal fortgeschrittene Erkrankungen als auch bei Vorliegen eines Rezidivs oder von Fernmetastasen. Die Leitlinie soll neben dem Beitrag für eine angemessene Gesundheitsversorgung auch die Basis für eine individuell zugeschnittene, qualitativ hochwertige und kosteneffiziente Therapie bieten. Mittel- und langfristig sollen so die Morbidität und Mortalität von Patienten mit hepatobiliären Tumoren gesenkt und die Lebensqualität erhöht werden.

\subsubsection{Adressaten}

Die Anwenderzielgruppe sind Ärztinnen und Ärzte aller Versorgungsbereiche (insbesondere Fachärztinnen und Fachärzte), die Patienten mit chronischen Lebererkrankungen, Verdacht auf sowie diagnostizierten hepatobiliären Tumoren ambulant und/ oder stationär behandeln. Hierzu gehören unter anderem Allgemeinmediziner, Internisten, Gastroenterologen und Hepatologen, Onkologen, Radiologen, Chirurgen, Palliativmediziner, Pathologen, Nuklearmediziner und Strahlentherapeuten, Psychoonkologen, onkologisch tätige Pflegekräfte und Physiotherapeuten sowie alle an einem HCC oder biliären Karzinom erkrankten Patienten und deren Angehörige. Weitere Adressaten dieser Leitlinie 
sind übergeordnete Organisationen (z. B. Krankenkassen). Sie soll entsprechend der Definition einer Leitlinie Entscheidungshilfen geben, jedoch keine Richtlinie sein. Der behandelnde Arzt ist weiterhin verpflichtet, unter Würdigung der Gesamtsituation des Patienten und mit diesem gemeinsam, die für die individuelle Situation angemessene Vorgehensweise zu finden.

\subsubsection{Gültigkeitsdauer und Aktualisierungsverfahren}

Die S3-Leitlinie ist bis zur nächsten Aktualisierung gültig, die Gültigkeitsdauer wird auf drei Jahre geschätzt. Vorgesehen sind regelmäßige Aktualisierungen, bei dringendem Änderungsbedarf werden diese gesondert publiziert. Kommentare und Hinweise für den Aktualisierungsprozess sind ausdrücklich erwünscht und können an die folgende Adresse gesendet werden: hcc@leitlinien programm-onkologie.de.

\subsubsection{Grundlagen der Methodik}

Die methodische Vorgehensweise bei der Erstellung der Leitlinie ist im Leitlinienreport dargelegt. Dieser ist im Internet z. B. auf den Seiten des Leitlinienprogramms Onkologie (http://leitlinien programm-onkologie.de/Leitlinien.7.0.html) und den Seiten der AWMF (http://www.awmf.org/) frei verfügbar.

\subsubsection{Schema der Evidenzgraduierung nach Oxford (Version 2011)}

Zur Klassifikation des Verzerrungsrisikos der identifizierten Studien wurde in dieser Leitlinie das in $>$ Tab. 4 aufgeführte System des Oxford Center for Evidence-based Medicine in der Version von 2011 verwendet. Dieses System sieht die Klassifikation der Studien für verschiedene klinische Fragestellungen (Nutzen von Therapie, prognostische Aussagekraft, diagnostische Wertigkeit) vor.

\subsubsection{Schema der Empfehlungsgraduierung}

Die Methodik des Leitlinienprogramms Onkologie sieht eine Vergabe von Empfehlungsgraden durch die Leitlinienautoren im Rahmen eines formalen Konsensusverfahrens vor. Dementsprechend wurden durch die AWMF moderierte, nominale Gruppenprozesse bzw. strukturierte Konsensuskonferenzen durchgeführt [2]. Im Rahmen dieser Prozesse wurden die Empfehlungen von den stimmberechtigten Mandatsträgern (siehe Kapitel 1.9.2) formal abgestimmt. Die Ergebnisse der jeweiligen Abstimmungen (Konsensstärke) sind entsprechend den Kategorien in Tab. 6 den Empfehlungen zugeordnet.

In der Leitlinie werden zu allen evidenzbasierten Statements (siehe Kapitel 2.2.3) und Empfehlungen das Evidenzlevel der zugrunde liegenden Studien sowie bei Empfehlungen zusätzlich die Stärke der Empfehlung (Empfehlungsgrad) ausgewiesen. Hinsichtlich der Stärke der Empfehlung werden in dieser Leitlinie drei Empfehlungsgrade unterschieden (siehe $>$ Tab. 5), die sich auch in der Formulierung der Empfehlungen jeweils widerspiegeln.

Die Entscheidungskriterien für die Festlegung der Empfehlungsgrade werden im Leitlinienreport zu dieser Leitlinie erläutert.

\subsubsection{Statements}

Als Statements werden Darlegungen oder Erläuterungen von spezifischen Sachverhalten oder Fragestellungen ohne unmittelbare Handlungsaufforderung bezeichnet. Sie werden entsprechend der Vorgehensweise bei den Empfehlungen im Rahmen eines formalen Konsensusverfahrens verabschiedet und können entweder auf Studienergebnissen oder auf Expertenmeinungen beruhen.

\subsubsection{Expertenkonsens (EK)}

Statements/Empfehlungen, für die eine Bearbeitung auf der Grundlage von Expertenkonsens der Leitliniengruppe beschlossen wurde, sind als Expertenkonsens ausgewiesen. Für diese Empfehlungen wurde keine systematische Literaturrecherche durchgeführt (die in den Hintergrundtexten ggf. angeführten Studien wurden von den beteiligten Fachexperten ausgewählt). Bei Empfehlungen, die auf einem Expertenkonsens basieren, werden keine Symbole bzw. Buchstaben verwendet, um die Empfehlungsstärke und die Qualität der Evidenz darzustellen. Die Stärke der Empfehlung ergibt sich hier allein aus der verwendeten Formulierung (soll/sollte/kann) entsprechend der Abstufung in $>$ Tab. 5 .

\subsubsection{Klug entscheiden}

Empfehlungen, die mit „Klug entscheiden“ gekennzeichnet sind, wurden für die „Klug entscheiden“-Initiative der Deutschen Gesellschaft für Innere Medizin ausgewählt. Diese Empfehlungen sollen als konkrete Hilfestellung bei der Indikationsstellung zu diagnostischen und therapeutischen Maßnahmen dienen, um eine Unter- bzw. Überversorgung zu vermeiden. Weitere Informationen finden Sie unter https://www.klug-entscheiden.com/.

\subsubsection{Unabhängigkeit und Darlegung möglicher Interessenkonflikte}

Die Deutsche Krebshilfe stellte die finanziellen Mittel über das Leitlinienprogramm Onkologie (OL) zur Verfügung. Diese Mittel wurden eingesetzt für Personalkosten, Büromaterial, Literaturbeschaffung und die Konsensuskonferenzen (Raummieten, Technik, Verpflegung, Moderatorenhonorare, Reisekosten der Teilnehmer). Die Erarbeitung der Leitlinie erfolgte in redaktioneller Unabhängigkeit von der finanzierenden Organisation. Alle Mitglieder legten während des Leitlinienprozesses mittels des AWMF-Formblatts eine schriftliche Erklärung zu eventuell bestehenden Interessenkonflikten (zu Beginn, Aktualisierung vor der Konsenskonferenz) vor. Die Interessenerklärungen sind im Leitlinienreport zu dieser Leitlinie (https://www.leitlinienprogramm-onkologie.de/ leitlinien/hepatozellulaeres-karzinom-hcc/) aufgeführt.

Der Umgang mit Interessenkonflikten wurde analog der Vorgaben der AWMF gemeinsam in unserem Steuergruppentreffen vom 18.07.2019 konsentiert. Zusätzlich wurde dieses Vorgehen mit Vertretern der DKG und der AWMF besprochen. Hier wurde folgendes Vorgehen festgehalten: 


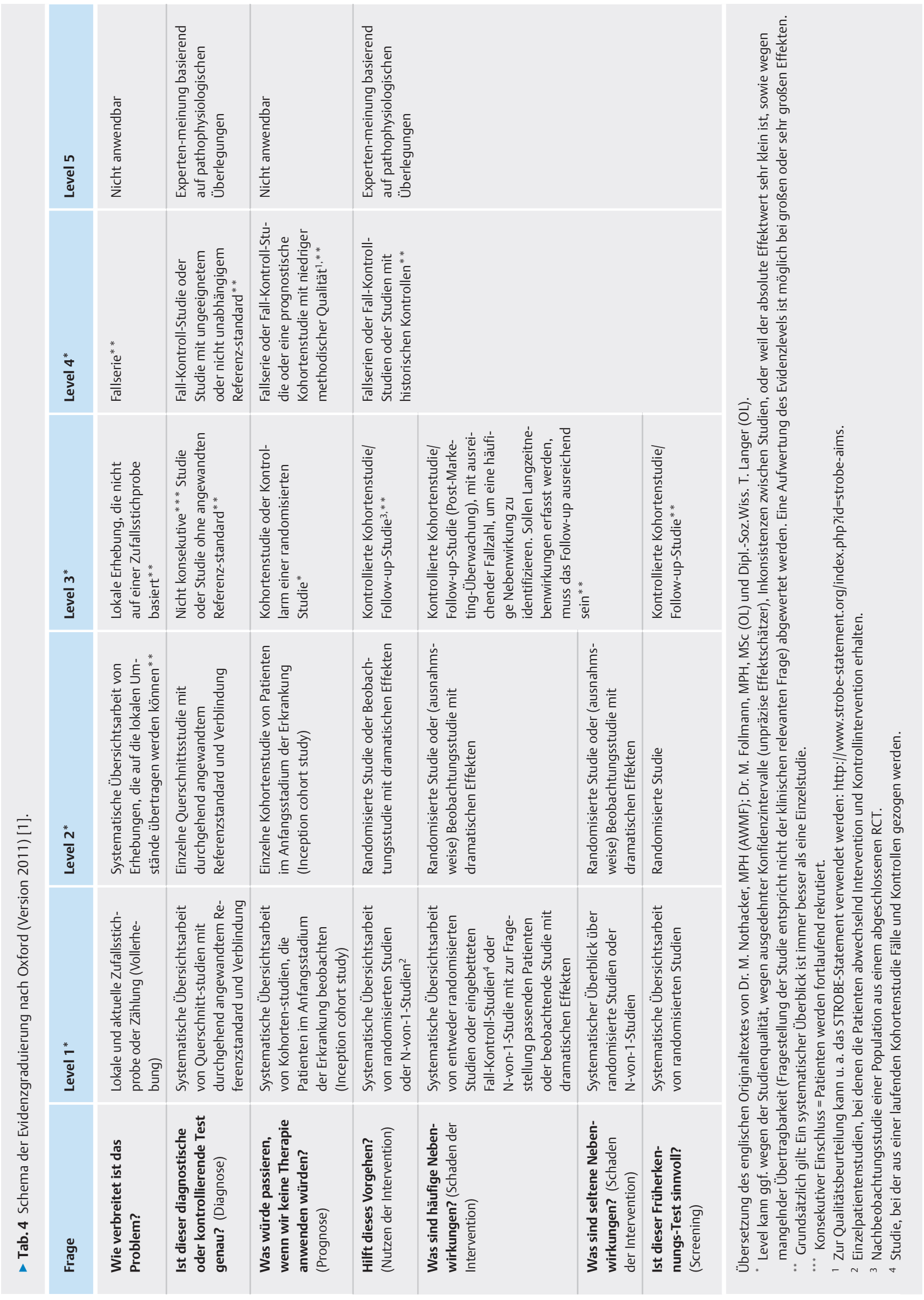


- Tab. 5 Schema der Empfehlungsgraduierung.

\begin{tabular}{|l|l|l|}
\hline Empfehlungsgrad & Beschreibung & Ausdrucksweise \\
\hline A & Starke Empfehlung & soll \\
\hline $\mathbf{B}$ & Empfehlung & sollte \\
\hline $\mathbf{0}$ & Empfehlung offen & kann \\
\hline
\end{tabular}

> Tab. 6 Konsensstärke.

\begin{tabular}{|l|l|}
\hline Konsensstärke & Prozentuale Zustimmung \\
\hline Starker Konsens & $>95 \%$ der Stimmberechtigten \\
\hline Konsens & $>75-95 \%$ der Stimmberechtigten \\
\hline Mehrheitliche Zustimmung & $50-75 \%$ der Stimmberechtigten \\
\hline Dissens & $<50 \%$ der Stimmberechtigten \\
\hline
\end{tabular}

- Es wurden alle direkten finanziellen und indirekten sekundären Interessen der letzten drei Jahre im Formular der Interessenkonflikte angegeben. Unmittelbar vor den beiden Konsensuskonferenzen erfolgte eine Aktualisierung der Erklärung.

- Entscheidend für die Bewertung war der thematische Bezug zur Leitlinie.

- Die Erklärungen zu den Interessenkonflikten werden im Leitlinienreport aufgeführt.

Umgang mit direkten finanziellen Interessenkonflikten:

- Es wurde bei finanziellen Vergütungen durch Ad-Board, Beratertätigkeit, Vortragstätigkeit und Industriedrittmitteln in verantwortlicher Position ein finanzieller Rahmen von $5000 € / J a h r$ und Firma festgesetzt. Oberhalb dieser Grenze kam es zur Feststellung eines moderaten Interessenkonfliktes.

- Wurde ein moderater Interessenkonflikt festgestellt, enthielt sich der Mandatsträger bei einzelnen Fragen oder Themenbereichen, je nach festgestelltem Interessenkonflikt.
- Im Hinblick auf die AG-Leiter wurde darauf geachtet, dass einem Mitglied der Steuergruppe mit einem moderateren Interessenkonflikt ein weiteres Mitglied der Steuergruppe mit keinem oder lediglich geringem Interessenkonflikt zur Seite gestellt wurde.

- Bei zwei Mandatsträgern wurden Patente festgestellt. Diese haben jedoch keinen thematischen Bezug zur Leitlinie, noch sind diese kommerzialisiert. Es erfolgte daher kein Ausschluss von der Leitlinienarbeit.

- Ein Aktienbesitz lag bei keinem Mandatsträger vor.

Umgang mit indirekten sekundären Interessenkonflikten:

- Mitgliedschaften in Fachgesellschaften und Beteiligung an Fortbildungen und Ausbildungsinstituten wurden nicht als Interessenkonflikt für diese Leitlinie bewertet, da diese ein essenzieller Teil der wissenschaftlichen und klinischen Arbeit sind.

- Ebenso wurde der Schwerpunkt der wissenschaftlichen und klinischen Tätigkeit in diesem Feld erwartet, um eine wissenschaftliche und praktikable Leitlinie zu erstellen.

- Eine persönliche Beziehung (Partner oder Verwandter 1. Grades) zu einem Vertretungsberechtigten eines Unternehmens der Gesundheitswirtschaft lag bei keinem Mandatsträger vor.

Der Vorschlag der Steuergruppe zum Management wurde zu Beginn der Konsenskonferenz diskutiert und umgesetzt.

Als protektive Faktoren gegen eine Verzerrung durch Interessenkonflikte können die systematische Evidenzaufbereitung, die pluralistische Zusammensetzung der Leitliniengruppe, die neutrale Moderation, die Diskussion der Bewertung der Interessen und des Umgangs mit Interessenkonflikten zu Beginn der Konsenskonferenz sowie die öffentliche Konsultation gewertet werden.

An dieser Stelle möchten wir allen Mitarbeitern für ihre ausschließlich ehrenamtliche Mitarbeit an dem Projekt danken.

\section{Diagnostik und Thearpie des hepatozellulären Karzinoms}

Die S3-Leitlinie Diagnostik und Therapie des hepatozellulären Karzinoms und biliärer Karzinome umfasst zwei Tumorentitäten. Zur Vereinfachung sind die Tumorentitäten hier getrennt veröffentlicht. An dieser Stelle verweisen wir daher auf die S3-Leitlinie Diagnostik und Therapie des hepatozellulären Karzinoms. 


\section{Diagnostik und Therapie der biliären Karzinome}

\subsection{Risikofaktoren, Prävention und Früherkennung}

\subsubsection{Risikofaktoren}

\begin{tabular}{|c|c|c|c|c|}
\hline Nr. & Empfehlung/Statement & EG & LoE & Quellen \\
\hline 4.1. & $\begin{array}{l}\text { Risikofaktoren für die Entwicklung eines intra- oder extrahepatischen } \\
\text { Cholangiokarzinoms sind: } \\
\text { - Adipositas } \\
\text { - Alkoholabusus } \\
\text { - Choledochus-Zysten } \\
\text { - Cholelithiasis } \\
\text { - Chronisch bakterielle Cholangitis } \\
\text { - Chronisch entzündliche Darmerkrankungen } \\
\text { - Chronische Hepatitis B-Virusinfektion } \\
\text { - Chronische Hepatitis C-Virusinfektion } \\
\text { - Diabetes mellitus } \\
\text { - Leberegel } \\
\text { - Leberzirrhose } \\
\text { - Nichtalkolische Fettlebererkrankung } \\
\text { - Parasitäre Cholangitiden } \\
\text { - Primär sklerosierende Cholangitis } \\
\text { - Rauchen } \\
\text { - Rezidivierende pyogene Cholangitiden }\end{array}$ & & $2-3$ & $\begin{array}{l}\text { De Novo } \\
\text { [3-9] }\end{array}$ \\
\hline 4.2. & $\begin{array}{l}\text { Risikofaktoren für die Entwicklung eines Gallenblasenkarzinoms sind: } \\
\text { - Anatomische Anomalien der intra- und extrahepatischen Gallenwege } \\
\text { - Cholelithiasis } \\
\text { - Chronisch bakterielle und parasitäre Cholangitis } \\
\text { - Diabetes mellitus } \\
\text { - Gallenblasenpolypen } \\
\text { - Porzellangallenblase } \\
\text { - Primär sklerosierende Cholangitis }\end{array}$ & & $2-3$ & $\begin{array}{l}\text { De Novo } \\
{[3,4,7,8,10,11]}\end{array}$ \\
\hline
\end{tabular}

4.1.2. Prophylaktische und therapeutische Maßnahmen zur Reduktion des Risikos der Entstehung von biliären Karzinomen

\begin{tabular}{|c|c|c|c|c|}
\hline Nr. & Empfehlung/Statement & EG & LoE & Quellen \\
\hline 4.3. & $\begin{array}{l}\text { Gallenblasenpolypen bei Patienten mit PSC sollten regelmäßig sonografisch } \\
\text { überwacht werden. In allen Fällen sollte die Indikation zur Cholezystektomie } \\
\text { diskutiert werden, bei Polypen über } 8 \text { mm oder Größenprogredienz sollte auf- } \\
\text { grund des erhöhten Karzinomrisikos unter Berücksichtigung der Leberfunktion } \\
\text { eine Cholezystektomie erfolgen. }\end{array}$ & EK & & \\
\hline 4.4. & $\begin{array}{l}\text { Patienten mit Gallenblasenpolypen } \geq 10 \text { mm sollte unabhängig von der } \\
\text { Symptomatik eine Cholezystektomie angeboten werden. }\end{array}$ & EK & & \\
\hline 4.5. & $\begin{array}{l}\text { Bei Patienten ohne Risikofaktoren* für ein Gallenblasenkarzinom mit Nachweis } \\
\text { eines Gallenblasenpolypen von <9 mm sollte eine sonografische Kontrolle in } \\
6 \text { Monaten (Polyp 6-9 mm) bzw. } 12 \text { Monaten (Polyp<6 mm) erfolgen. } \\
{ }^{*} \text { Risikofaktoren für neoplastische Polypen: Alter > } 50 \text { Jahre, bekannte PSC, } \\
\text { Zugehörigkeit zu einer indigenen Population oder Vorhandensein eines sessilen } \\
\text { Polypen [12] }\end{array}$ & EK & & \\
\hline
\end{tabular}




\subsubsection{Früherkennung}

\begin{tabular}{|l|l|l|l|l|l|}
\hline Nr. & Empfehlung/Statement & EG & Quellen \\
\hline 4.6. & $\begin{array}{l}\text { Bei Patienten mit PSC sollte halbjährlich eine Bildgebung im Rahmen der } \\
\text { Cholangiokarzinom-Füherkennung durchgeführt werden. }\end{array}$ & EK \\
\hline
\end{tabular}

\subsection{Histopathologische und molekulare Diagnostik}

Nr.

4.7.

4.8.

4.9

4.10.

4.11.

\section{Empfehlung/Statement}

Vor oder im Rahmen einer Tumortherapie sollen Tumoren der Gallenwege und Gallenblase histologisch oder ggf. zytologisch gesichert werden.

Die Typisierung der Karzinome der Gallenwege und der Gallenblase soll nach der anatomischen Lokalisation (intrahepatisch, perihilär, distale Gallenwege, Gallenblase) und gemäß der histologischen Differenzierung nach der aktuellen WHOKlassifikation erfolgen. Bei intrahepatischen Cholangiokarzinomen sollte eine Unterscheidung des Typs „small duct“ und „large duct“ erfolgen.

9. Die Bearbeitung und Befundung eines Resektats soll die Ausdehnung des Tumors (Staging) gemäß der aktuellen TNM-Klassifikation, seinen Typ (Typing) und Differenzierungsgrad (Grading) und den Status des Resektatrandes (R-Klassifikation) sowie bei intrahepatischen Cholangiokarzinomen den Status der nichttumorösen Leber ermitteln. Bei Präparaten mit prämalignen Läsionen soll durch genaue Aufarbeitung ein möglicher Übergang in ein invasives Karzinom abgeklärt werden.

Die Diagnose eines Cholangiokarzinoms kann bei klarer Konstellation durch die konventionelle Histologie gestellt werden.

In unklaren Fällen, insbesondere bei intrahepatischen Tumoren, soll die Diagnose durch geeignete immunhistologische und oder molekularpathologische Untersuchungen abgesichert werden.

Cholangiokarzinome weisen potenzielle Zielstrukturen für eine molekular gesteuerte Systemtherapie auf, die im geeigneten Kontext getestet werden sollten.
EG

LoE

Quellen

EK

EK

EK

EK

EK

Zulassung

\begin{tabular}{|l|l|l|}
\hline Molekulare Alteration & Häufigkeit (\%) & Zulassung \\
\hline RAS-Mutation & $10-20$ & \\
\hline TP53-Mutation & $20-30$ & \\
\hline FGFR2-Translokation & $15-30$ & Zulassung \\
\hline IDH1/2 & $10-20$ & Zulassung außerhalb der EU \\
\hline ARID1A & $5-15$ & \\
\hline BAP1 & $5-15$ & \\
\hline BRAF V600E & $3-6$ & Zulassung bei anderer Entität \\
\hline ERBB2 & $2-3$ & Zulassung bei anderer Entität \\
\hline MSI-H (MLH1, MSH2, MSH6, PMS2) & $1-2$ & Zulassung außerhalb der EU \\
\hline NTRK1-3 & $<1$ & Zulassung \\
\hline NRG1 & $<1$ & Zulassung bei anderer Entität \\
\hline
\end{tabular}




\subsection{Bildgebende und endoskopische Diagnostik}

\begin{tabular}{|c|c|c|c|c|}
\hline Nr. & Empfehlung/Statement & EG & LoE & Quellen \\
\hline 4.12. & $\begin{array}{l}\text { Die Sonografie wird häufig zur initialen Einschätzung bei V. a. auf ein biliäres Kar- } \\
\text { zinom verwendet. }\end{array}$ & \multicolumn{3}{|l|}{ EK } \\
\hline 4.13. & $\begin{array}{l}\text { Zur initialen Diagnostik und zum Staging bei kurativer Intention eines Cholangio- } \\
\text { karzinoms sollen eine mehrphasische hepatische MRT-Untersuchung sowie ein } \\
\text { kontrastverstärktes CT des Thorax und des Abdomens* vorliegen. } \\
\text { * Wenn komplettes MRT-Abdomen vorliegt, muss kein CT des Abdomens ergänzt } \\
\text { werden. }\end{array}$ & A & 1 & $\begin{array}{l}\text { De Novo } \\
{[13]}\end{array}$ \\
\hline 4.14. & $\begin{array}{l}\text { Für die Erfassung der maximalen Ausbreitung des Tumors inklusive Gefäßinvasion } \\
\text { soll, wenn eine kurative Behandlungsoption besteht, mindestens ein dynamisches } \\
\text { kontrastverstärktes MRT eingesetzt werden. }\end{array}$ & \multicolumn{3}{|l|}{ EK } \\
\hline 4.15. & $\begin{array}{l}\text { Bei Verdachtsdiagnose auf ein Cholangiokarzinom soll eine Schnittbildgebung zur } \\
\text { Beurteilung der Tumorausdehnung verwendet werden. }\end{array}$ & A & 1 & $\begin{array}{l}\text { De Novo } \\
{[13]}\end{array}$ \\
\hline \multicolumn{5}{|c|}{ 4.3.1. Endoskopische Diagnostik } \\
\hline Nr. & Empfehlung/Statement & EG & LoE & Quellen \\
\hline 4.16. & $\begin{array}{l}\text { Der endoskopische Ultraschall kann zur Diagnose, lokalem Staging und } \\
\text { Gewebegewinnung beim biliären Karzinom verwendet werden. }\end{array}$ & 0 & 1 & $\begin{array}{l}\text { De Novo } \\
{[14]}\end{array}$ \\
\hline 4.17. & $\begin{array}{l}\text { Wenn im Rahmen einer ERCP der V. a. ein extrahepatisches Cholangiokarzinom } \\
\text { gestellt wird, sollte im Rahmen einer ERCP eine Zangenbiospie oder eine Bürs- } \\
\text { tenzytologie entnommen werden. }\end{array}$ & EK & & \\
\hline 4.18. & $\begin{array}{l}\text { Bei V. a. ein extrahepatisches Cholangiokarzinom kann durch Einsatz der Cholan- } \\
\text { gioskopie in Kombination mit visuell gezielter Biopsie die Sensitivität der Diagnose } \\
\text { gesteigert werden. }\end{array}$ & & 1 & $\begin{array}{l}\text { De Novo } \\
{[15]}\end{array}$ \\
\hline 4.19. & $\begin{array}{l}\text { Patienten mit primär sklerosierender Cholangitis und Erstmanifestation einer } \\
\text { dominanten Stenose sollen mittels MRT/MRCP und ERCP/Histologie weiter a- } \\
\text { bgeklärt werden. } \\
\text { Bei weiter bestehendem Verdacht auf ein CCA trotz negativer Diagnostik sollte eine } \\
\text { kurzfristige erneute Reevaluation, ggf. mit Wiederholung der Untersuchungen, } \\
\text { oder bei therapeutischer Relevanz eine Klärung im Rahmen einer explorativen } \\
\text { Laparotomie erfolgen. }\end{array}$ & EK & & \\
\hline
\end{tabular}

\subsection{Operative und interventionelle Therapieverfahren}

\subsubsection{Resektion}

\begin{tabular}{|c|c|c|c|c|}
\hline Nr. & Empfehlung/Statement & EG & LoE & Quellen \\
\hline 4.20. & $\begin{array}{l}\text { Eine Resektion eines pCCA, dCCA oder iCCA soll erfolgen, wenn eine komplette } \\
\text { Resektion (R0-Resektion) möglich erscheint. }\end{array}$ & EK & & \\
\hline 4.21. & $\begin{array}{l}\text { Eine Resektion eines Gallenblasenkarzinoms soll erfolgen, wenn klinisch keine } \\
\text { Fernmetastasen vorliegen (cM0) und eine komplette Resektion (RO-Resektion) } \\
\text { möglich erscheint. }\end{array}$ & EK & & \\
\hline 4.22. & $\begin{array}{l}\text { Werden bei einer Cholezystektomie intra- oder postoperativ ein Carcinoma in situ } \\
\text { (Tis) oder ein Mukosakarzinom (T1a) festgestellt, sollte bei Vorliegen einer } \\
\text { R0-Situation (D. cysticus) keine Nachresektion erfolgen. }\end{array}$ & EK & & \\
\hline 4.23 . & $\begin{array}{l}\text { Bei intra- oder postoperativem Nachweis eines Gallenblasenkarzinoms der Kate- } \\
\text { gorie } \geq T 1 \text { b soll bei kurativem Ansatz eine onkologische Resektion oder Nachre- } \\
\text { sektion erfolgen. }\end{array}$ & EK & & \\
\hline 4.24. & $\begin{array}{l}\text { Bei einem isolierten intrahepatischen Rezidiv eines CCA kann eine erneute Re- } \\
\text { sektion durchgeführt werden, wenn eine komplette Resektion (R0-Resektion) } \\
\text { möglich erscheint. }\end{array}$ & EK & & \\
\hline
\end{tabular}


Nr.

4.25 .

\section{Empfehlung/Statement}

Wenn Rezidive nach einer vorangegangenen Operation nicht erneut operativ versorgt werden können, können diese mit thermischer Ablation behandelt werden, wenn hierdurch eine komplette Ablation möglich erscheint.
EG

LoE

Quellen

EK

\subsubsection{Lebertransplantation}

\begin{tabular}{|c||c|c|c|c|c|}
\hline Nr. & Empfehlung/Statement & LG & LoE \\
\hline 4.26. & Eine Lebertransplantation für das iCCA soll außerhalb von Studien nicht erfolgen. & EK \\
\hline 4.27. & $\begin{array}{l}\text { Bei irresektablem, nichtmetastasiertem pCCA, welches die Mayo-Kriterien erfült, } \\
\text { kann eine Lebertransplantation unter Studienbedingungen erwogen werden. }\end{array}$ & 0 \\
\hline
\end{tabular}

\section{Mayo-Kriterien}

Irresektables pCCA oder pCCA in PSC-Zirrhose

Tumor-Durchmesser $<3 \mathrm{~cm}$

keine LK-Metastasen (obligate chirurgische Exploration)

keine extrahepatische Tumormanifestation

histologisch/zytologisch bestätigtes pCCA oder CA19-9>1000 kU/L mit Vorliegen radiologischer Zeichen einer malignen Stenose

\subsubsection{Interventionelle Therapieverfahren}
Nr.

\section{Empfehlung/Statement}
4.28. Lokale Verfahren (RFA/MWA) können nach Beschluss des Tumorboards durchgeführt werden, falls keine Resektion möglich ist.
4.29. Beim fortgeschrittenen iCCA ohne extrahepatischen Befall können intraarterielle Verfahren ab der Zweitlinie oder additiv zur Chemotherapie, nach Vorstellung im Tumorboard, erfolgen.

\begin{tabular}{|l|l|l|}
\hline EG & LoE & Quellen \\
\hline EK & \\
\hline EK
\end{tabular}

\subsubsection{Endoskopische Therapieverfahren}

\begin{tabular}{|c|l|l|l|}
\hline Nr. & Empfehlung/Statement & EG & Luellen \\
\hline 4.30. & $\begin{array}{l}\text { Die Indikation für eine präoperative biliäre Drainage sollte interdisziplinär } \\
\text { getroffen werden. }\end{array}$ & EK, Klug entscheiden \\
\hline 4.31. & $\begin{array}{l}\text { Bei Vorliegen einer Cholangitis sollte eine präoperative biliäre Drainage } \\
\text { umgehend erfolgen. }\end{array}$ & EK, Klug entscheiden \\
\hline 4.32. & $\begin{array}{l}\text { Eine palliative biliäre Drainage soll Patienten mit symptomatischem } \\
\text { Gallenwegsverschluss angeboten werden. }\end{array}$ & EK \\
\hline 4.33. & $\begin{array}{l}\text { Eine palliative Drainage sollte in einem erfahrenen Zentrum durchgeführt } \\
\text { werden, da hier auch alternative Drainageverfahren zur Verfügung stehen. }\end{array}$ \\
\hline 4.34. & $\begin{array}{l}\text { Intraduktale, lokalablative Verfahren (fotodynamische Therapie und intraduktale } \\
\text { RFA) können nach Beschluss des Tumorboards durchgeführt werden, um eine } \\
\text { effektive Palliation zu ermöglichen. }\end{array}$ & EK \\
\hline
\end{tabular}




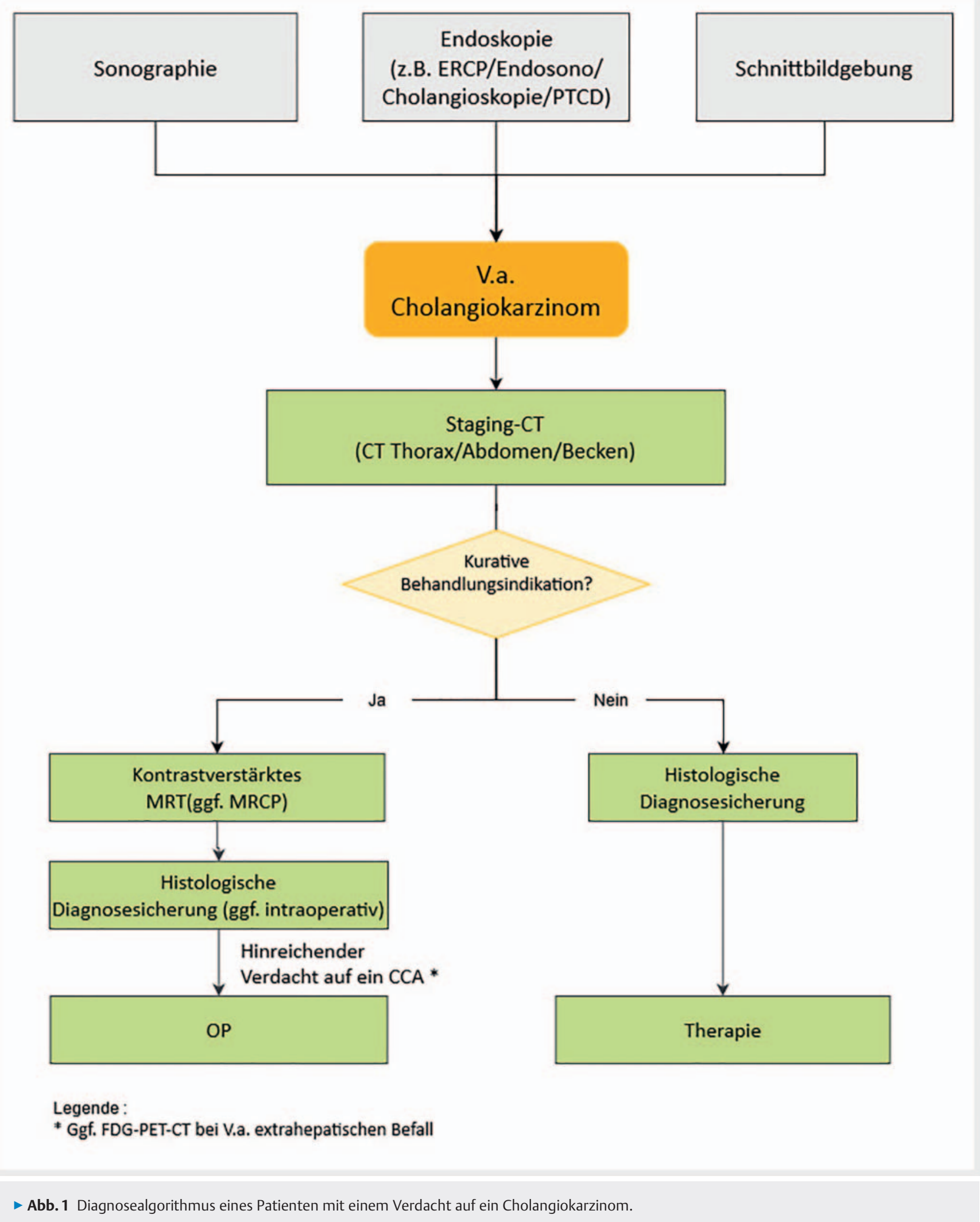




\subsubsection{Stereotaxie}

\begin{tabular}{|l|l|l|l|l|l|}
\hline Nr. & Empfehlung/Statement & LG & Luellen \\
\hline 4.35. & $\begin{array}{l}\text { Eine Hochpräzisionsradiotherapie (Stereotactic Body Radiotherapy; SBRT) kann } \\
\text { nach Beschluss in einer Tumorkonferenz bei fehlenden alternativen Therapie- } \\
\text { optionen angeboten werden. }\end{array}$ & EK \\
\hline
\end{tabular}

\subsubsection{Nachsorge}

\begin{tabular}{l|l}
\hline Nr. & Empfehlung/Statement \\
\hline $\begin{array}{l}\text { 4.36. } \\
\text { Nach Resektion/Ablation eines CCA sollte nach 4-12 Wochen erstmals, im ersten } \\
\text { Jahr alle 3 Monate und im zweiten Jahr alle 3-6 Monate ein biphasisches CT oder } \\
\text { ein dynamisches MRT durchgeführt werden. }\end{array}$ \\
\hline
\end{tabular}

\begin{tabular}{l|l|l} 
EG & LoE & Quellen \\
\hline
\end{tabular}

EK

\subsection{Systemtherapie}

4.5.1. Adjuvante Therapie

\begin{tabular}{|c|c|c|c|c|}
\hline Nr. & Empfehlung/Statement & EG & LoE & Quellen \\
\hline 4.37. & $\begin{array}{l}\text { Nach chirurgischer Tumorentfernung besteht ein Rezidivrisiko von } 40-80 \% \text {. Insbe- } \\
\text { sondere Patienten mit Lymphknotenmetastasen (N1), positiven Resektionsrändern } \\
\text { (R1) oder niedrigem Differenzierungsgrad (G3) haben ein hohes Rezidivrisiko. }\end{array}$ & EK & & \\
\hline 4.38. & $\begin{array}{l}\text { Aufgrund des hohen Rezidivrisikos sollten Patienten nach chirurgischer Tumor- } \\
\text { entfernung (R0, R1) eine adjuvante Systemtherapie mit Capecitabin angeboten } \\
\text { werden. }\end{array}$ & B & 2 & $\begin{array}{l}\text { De Novo } \\
{[18]}\end{array}$ \\
\hline
\end{tabular}

4.5.2. Neoadjuvante Therapie primär resektabler Tumoren

\begin{tabular}{|l|l|l|l|l|l|}
\hline Nr. & Empfehlung/Statement & QG & Luellen \\
\hline 4.39. & $\begin{array}{l}\text { Eine neoadjuvante Chemotherapie soll bei primär resektablen Tumoren nicht } \\
\text { außerhalb von klinischen Studien erfolgen. }\end{array}$ & EK \\
\hline
\end{tabular}

\subsubsection{Systemtherapie lokal fortgeschrittener Tumoren}

\begin{tabular}{|l|l|l|l|}
\hline Nr. & Empfehlung/Statement & LG & LoE \\
\hline 4.40. & $\begin{array}{l}\text { Bei primär irresektablen Tumoren sollte unter einer Tumortherapie eine erneute } \\
\text { Vorstellung im Tumorboard mit der Frage einer sekundären Resektabilität erfolgen. }\end{array}$ & EK \\
\hline
\end{tabular}




\subsubsection{Palliative Systemtherapie}

\begin{tabular}{|c|c|c|c|c|}
\hline Nr. & Empfehlung/Statement & EG & LoE & Quellen \\
\hline 4.41. & $\begin{array}{l}\text { Allen Patienten mit Cholangio- oder Gallenblasenkarzinom soll bei adäquatem } \\
\text { Allgemeinzustand in der inoperablen lokal fortgeschrittenen oder metastasierten } \\
\text { Situation eine palliative Systemtherapie angeboten werden. }\end{array}$ & A & 2 & \multirow[t]{2}{*}{$\begin{array}{l}\text { De Novo } \\
{[19-22]}\end{array}$} \\
\hline 4.42. & $\begin{array}{l}\text { Als Systemtherapie soll in der Erstlinie die Kombination Gemcitabin und Cisplatin } \\
\text { angeboten werden. }\end{array}$ & A & 2 & \\
\hline 4.43. & $\begin{array}{l}\text { Nach Versagen oder Unverträglichkeit der Erstlinientherapie soll Patienten mit } \\
\text { adäquatem Allgemeinzustand eine weitere Therapie angeboten werden. }\end{array}$ & \multicolumn{3}{|l|}{ EK } \\
\hline 4.44 & $\begin{array}{l}\text { Als medikamentöse Zweitlinientherapie kann eine Therapie mit FOLFOX } \\
\text { angeboten werden. }\end{array}$ & \multicolumn{3}{|l|}{ EK } \\
\hline 4.45 & $\begin{array}{l}\text { In einer palliativen Situation bei Patienten mit ECOG } 0 \text {-1 sollte eine molekulare } \\
\text { Charakterisierung des Tumors und Vorstellung in einem interdisziplinären/mole- } \\
\text { kularen Tumorboard erfolgen. }\end{array}$ & \multicolumn{3}{|c|}{ EK, Klug entscheiden } \\
\hline
\end{tabular}

\subsubsection{Verlaufskontrollen unter Systemtherapie}

\begin{tabular}{|l|l|l|l|l|}
\hline Nr. & Empfehlung/Statement & Quellen \\
\hline 4.46. & $\begin{array}{l}\text { Bei biliären Karzinomen unter Systemtherapie sollte alle 6-12 Wochen die } \\
\text { diagnostisch am besten geeignete Schnittbildgebung durchgeführt werden. } \\
\text { Die Interpretation im klinischen Alltag sollte sich an den Auswerteprinzipien von } \\
\text { RECIST } 1.1 \text { orientieren. }\end{array}$ & EK \\
\hline
\end{tabular}

\subsection{Supportivtherapie des hepatozellulären Karzinoms und der biliären Karzinome}

\subsubsection{Ernährung}

$\mathrm{Nr}$

3.89 .
Empfehlung/Statement

Mangelernährung beeinträchtigt Lebensqualität und Therapietoleranz.

Eine Mangelernährung sollte erfasst und behandelt werden.
EC

LoE

Quellen

4.6.2. Palliativmedizinische Behandlung beim HCC/CCA

\begin{tabular}{|c|c|c|c|c|}
\hline Nr. & Empfehlung/Statement & EG & LoE & Quellen \\
\hline 3.90. & $\begin{array}{l}\text { Alle Patienten mit einer Krebserkrankung sollen unabhängig vom } \\
\text { Krankheitsstadium Zugang zu Informationen über Palliativversorgung } \\
\text { (z. B. durch Auslage von Flyern) haben. }\end{array}$ & EK & & \\
\hline 3.91. & $\begin{array}{l}\text { Allen Patienten soll nach der Diagnose einer nichtheilbaren Krebserkrankung eine } \\
\text { Palliativversorgung angeboten werden, unabhängig davon, ob eine tumorspezifi- } \\
\text { sche Therapie durchgeführt wird. }\end{array}$ & A & 1 & $\begin{array}{l}\text { S3-Leitlinie Palliativ- } \\
\text { medizin Langversion } \\
2.1 \text { - Januar } 2020\end{array}$ \\
\hline 3.92. & $\begin{array}{l}\text { Allen Patienten mit einem HCC im Stadium BCLC D sollte aktiv eine Palliativ- } \\
\text { versorgung angeboten werden. }\end{array}$ & EK & & \\
\hline 3.93. & $\begin{array}{l}\text { Allen Patienten mit einem Cholangiokarzinom im Stadium IV nach UICC-Klassifi- } \\
\text { kation soll eine Palliativversorgung angeboten werden. }\end{array}$ & EK & & \\
\hline 3.94. & Der Pruritus sollte analog der AWMF-S2k-Leitlinie Pruritus behandelt werden. & EK & & \\
\hline
\end{tabular}


4.6.3. Rehabilitation, Sport- und Bewegungstherapie

\begin{tabular}{|c|c|c|c|c|}
\hline Nr. & Empfehlung/Statement & EG & LoE & Quellen \\
\hline 3.95. & $\begin{array}{l}\text { Patienten mit HCC/CCA sollten zu körperlichen Aktivitäten und/oder } \\
\text { Bewegungstherapie motiviert werden. }\end{array}$ & EK & & \\
\hline 3.96. & $\begin{array}{l}\text { Der Nutzen einer Anschlussheilbehandlung und von Rehabilitationsmaßnahmen } \\
\text { (hinsichtlich Lebensqualität, Leistungsfähigkeit, krankheitsfreiem Überleben, Ge- } \\
\text { samtüberleben) ist für das HCC/CCA nicht ausreichend evaluiert. Dennoch sollte } \\
\text { den Patienten, die die Voraussetzungen erfüllen, eine AHB oder Rehabilitation } \\
\text { angeboten werden. }\end{array}$ & EK & & \\
\hline 3.97. & $\begin{array}{l}\text { Die rehabilitative Therapie soll medizinische, pflegerische, aufklärende, } \\
\text { trainierende und psychosoziale Maßnahmen umfassen, die dem individuellen } \\
\text { Rehabilitationsbedarf angepasst werden. }\end{array}$ & EK & & \\
\hline
\end{tabular}

4.6.4. Psychoonkologie

\begin{tabular}{|l|l|l|l|}
\hline Nr. & Empfehlung/Statement & EG & LoE \\
\hline 3.98. & $\begin{array}{l}\text { Die Erfassung der psychischen Belastung sowie die psychoonkologische Behand- } \\
\text { lung sollten, wie in der S3-Querschnittsleitlinie Psychoonkologie beschrieben, } \\
\text { erfolgen. }\end{array}$ & EK \\
\hline 3.99. & $\begin{array}{l}\text { Die Kommunikation mit Patienten mit HCC/CCA und ihren Angehörigen soll wie- } \\
\text { derholt in allen Phasen der Erkrankung und durch alle behandelnden Berufsgrup- } \\
\text { pen patientenzentriert erfolgen und soll sich an deren individuellen Anliegen, } \\
\text { Bedürfnissen und Präferenzen orientieren, welche Information, Aufklärung und } \\
\text { Beteiligung an Entscheidungen betreffen. }\end{array}$ & EK \\
\hline
\end{tabular}

\section{Qualitätsindikatoren}

Qualitätsindikatoren, die gemäß einer standardisierten Methodik von den starken (A) Empfehlungen dieser Leitlinie abgeleitet wurden, finden sich in der Langversion dieser Leitlinie.

\section{Abbildungsverzeichnis}

- Abb. 1: Diagnosealgorithmus eines Patienten mit einem Verdacht auf ein Cholangiokarzinom

\section{Tabellenverzeichnis}

\begin{tabular}{|c|c|}
\hline - Tab. 1: Beteiligte Fachgesellschaften und Organisationen & 223 \\
\hline Tab. 2: Arbeitsgruppen und deren Mitglieder & 224 \\
\hline - Tab. 3: Beteiligte Experten & 224 \\
\hline $\begin{array}{l}\text { Tab. 4: Schema der Evidenzgraduierung nach Oxford } \\
\text { (Version 2011) [1] }\end{array}$ & 228 \\
\hline - Tab. 5: Schema der Empfehlungsgraduierung & 229 \\
\hline - Tab. 6: Konsensstärke & 229 \\
\hline Tab. 7: Molekulare Alterationen beim small duct iCCA & 231 \\
\hline
\end{tabular}

Interessenkonflikt

Die Übersicht über die Interessenkonflikte der Autorinnen und Autoren sind im Leitlinienreport veröffentlicht.

\section{Literatur}

[1] Howick J et al. The 2011 Oxford CEBM Evidence Levels of Evidence (Introductory Document). 2011. Available from: http://www.cebm.net/index. aspx?o $=5653$

[2] Arbeitsgemeinschaft der Wissenschaftlichen Medizinischen Fachgesellschaften - Ständige Kommission, L. AWMF-Regelwerk „Leitlinien“. 1. Auflage 2012 [cited 09.12.2013. Available from: http://www.awmf. org/leitlinien/awmf-regelwerk/awmf-regelwerk.html

[3] Atchison EA et al. Risk of cancer in a large cohort of U.S. veterans with diabetes. Int J Cancer 2011; 128: 635-643

[4] de Valle MB, Björnsson E, Lindkvist B. Mortality and cancer risk related to primary sclerosing cholangitis in a Swedish population-based cohort. Liver Int 2012; 32: 441-448

[5] El-Serag HB et al. Risk of hepatobiliary and pancreatic cancers after hepatitis C virus infection: A population-based study of U.S. veterans. Hepatology 2009; 49: 116-123

[6] Huang $Y$ et al. Smoking and risk of cholangiocarcinoma: a systematic review and meta-analysis. Oncotarget 2017; 8: 100570-100581

[7] Jing $W$ et al. Diabetes mellitus and increased risk of cholangiocarcinoma: a meta-analysis. Eur J Cancer Prev 2012; 21: 24-31 
[8] Palmer WC, Patel T. Are common factors involved in the pathogenesis of primary liver cancers? A meta-analysis of risk factors for intrahepatic cholangiocarcinoma. J Hepatol 2012; 57: 69-76

[9] Wongjarupong $\mathrm{N}$ et al. Non-alcoholic fatty liver disease as a risk factor for cholangiocarcinoma: a systematic review and meta-analysis. BMC Gastroenterol 2017; 17: 149

[10] Park JY et al. Long-term follow up of gallbladder polyps. J Gastroenterol Hepatol 2009; 24: 219-222

[11] Nagaraja V, Eslick GD. Systematic review with meta-analysis: the relationship between chronic Salmonella typhi carrier status and gall-bladder cancer. Aliment Pharmacol Ther 2014; 39: 745-750

[12] Wiles R et al. Management and follow-up of gallbladder polyps: Joint guidelines between the European Society of Gastrointestinal and Abdominal Radiology (ESGAR), European Association for Endoscopic Surgery and other Interventional Techniques (EAES), International Society of Digestive Surgery - European Federation (EFISDS) and European Society of Gastrointestinal Endoscopy (ESGE). Eur Radiol 2017; 27: 3856-3866

[13] Zhang $\mathrm{H}$ et al. Radiological Imaging for Assessing the Respectability of Hilar Cholangiocarcinoma: A Systematic Review and Meta-Analysis. Biomed Res Int 2015; 2015: 497942

[14] De Moura DTH et al. Endoscopic retrograde cholangiopancreatography versus endoscopic ultrasound for tissue diagnosis of malignant biliary stricture: Systematic review and meta-analysis. Endosc Ultrasound 2018; 7: 10-19
[15] Navaneethan U et al. Single-operator cholangioscopy and targeted biopsies in the diagnosis of indeterminate biliary strictures: a systematic review. Gastrointest Endosc 2015; 82: 608-614 e2

[16] Becker NS et al. Outcomes analysis for 280 patients with cholangiocarcinoma treated with liver transplantation over an 18 -year period. J Gastrointest Surg 2008; 12: 117-122

[17] Darwish Murad S et al. Efficacy of neoadjuvant chemoradiation, followed by liver transplantation, for perihilar cholangiocarcinoma at 12 US centers. Gastroenterology 2012; 143: 88-98.e3, quiz e14

[18] Primrose JN et al. Capecitabine compared with observation in resected biliary tract cancer (BILCAP): a randomised, controlled, multicentre, phase 3 study. Lancet Oncol 2019; 20: 663-673

[19] Valle J et al. Cisplatin plus gemcitabine versus gemcitabine for biliary tract cancer. N Engl J Med 2010; 362: 1273-1281

[20] Okusaka T et al. Gemcitabine alone or in combination with cisplatin in patients with biliary tract cancer: a comparative multicentre study in Japan. Br J Cancer 2010; 103: 469-474

[21] Valle JW et al. Cisplatin and gemcitabine for advanced biliary tract cancer: a meta-analysis of two randomised trials. Ann Oncol 2014; 25: 391-398

[22] Park JO et al. Gemcitabine Plus Cisplatin for Advanced Biliary Tract Cancer: A Systematic Review. Cancer Res Treat 2015; 47: 343-361 